Provided for non-commercial research and educational use only. Not for reproduction or distribution or commercial use.

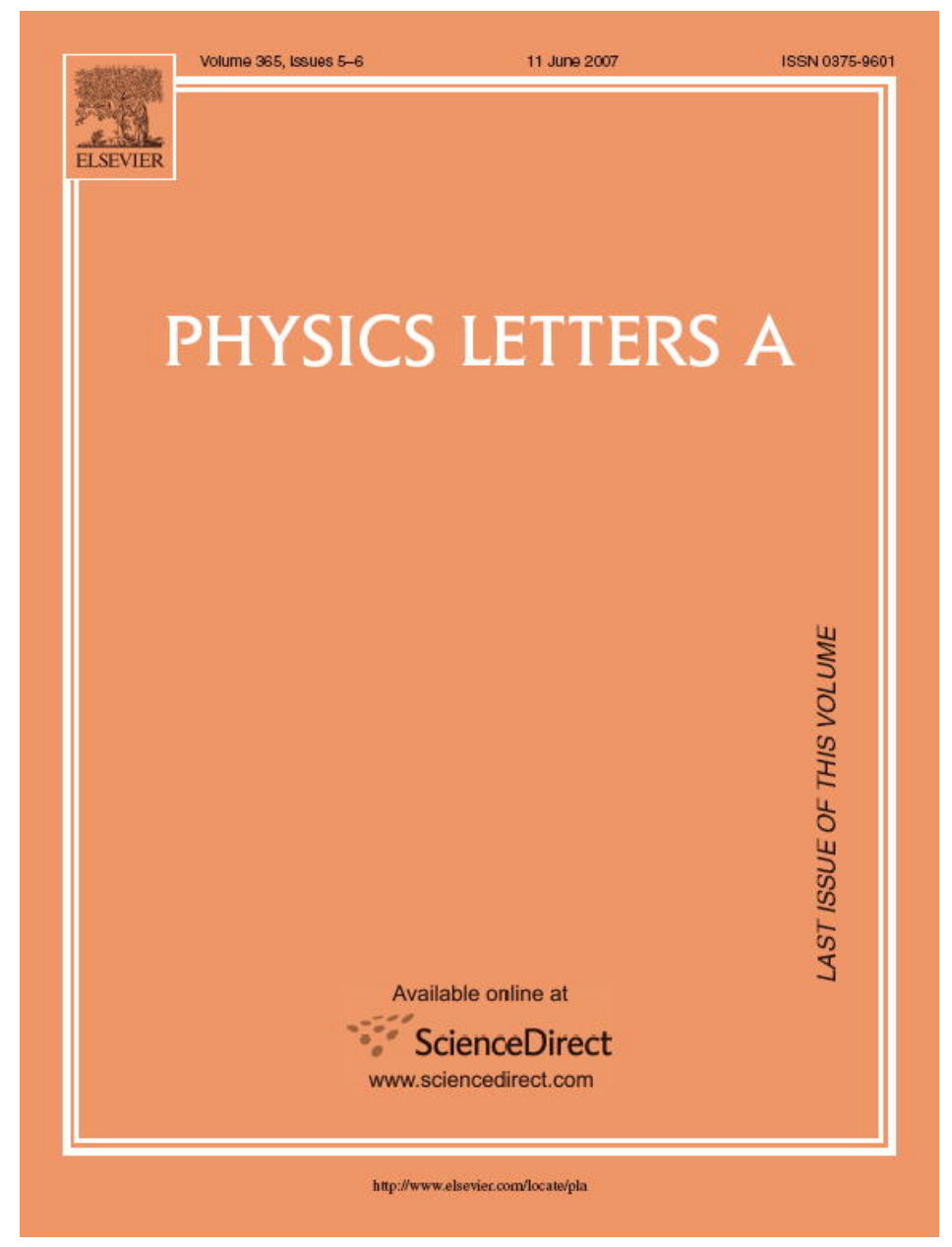

This article was originally published in a journal published by Elsevier, and the attached copy is provided by Elsevier for the author's benefit and for the benefit of the author's institution, for non-commercial research and educational use including without limitation use in instruction at your institution, sending it to specific colleagues that you know, and providing a copy to your institution's administrator.

All other uses, reproduction and distribution, including without limitation commercial reprints, selling or licensing copies or access,

or posting on open internet sites, your personal or institution's website or repository, are prohibited. For exceptions, permission may be sought for such use through Elsevier's permissions site at: 


\title{
Supersymmetry model of a binary mixture with noise of the diffusion flux
}

\author{
Alexander Olemskoi a, Peter Galenko ${ }^{\mathrm{b}, *}$, Anna Shmatko ${ }^{\mathrm{c}}$ \\ a Applied Physics Institute, 58, Petropavlovskaya street, 40030 Sumy, Ukraine \\ ${ }^{\mathrm{b}}$ Institute of Materials Physics in Space, German Aerospace Center, 51170 Cologne, Germany \\ ${ }^{\mathrm{c}}$ Kharkov National University, 4, Svoboda square, 61002 Kharkov, Ukraine
}

Received 21 September 2006; received in revised form 19 December 2006; accepted 20 December 2006

Available online 31 January 2007

Communicated by C.R. Doering

\begin{abstract}
Supersymmetry theory of diffusion is elaborated to study analytically a non-equilibrium binary mixture with relaxation of diffusion flux. Within such a scheme, Bose components represent order parameter and amplitude of fluctuations of the diffusion flux, while the mutually conjugated combination of Fermi-components gives the mixture concentration. For above variables, the system of equations is found to describe self-consistent behavior of a binary mixture, when the shortest observable time interval is longer than the flux relaxation time.
\end{abstract}

(c) 2007 Elsevier B.V. All rights reserved.

PACS: 05.10.Gg; 05.40.Ca; 05.70.Fh; 11.30.Pb; 12.60.Jv

Keywords: Supersymmetry theory; Diffusion; Relaxation; Flux; Concentration

\section{Introduction}

Non-equilibrium binary systems under diffusion transport were intensively investigated in past decades. Particularly, one of the main focuses of theoretical investigation was to describe non-equilibrium systems at high frequency or high speed of perturbations (see overviews [1,2] and references therein). Experimentally, non-equilibrium binary systems under diffusion may exist in rapidly quenched liquid mixtures, processes with shock waves, deeply supercooled phases, and, generally, in systems under rapid phase transformations. Using extended thermodynamical theory [1,2], a role of relaxation and inertial processes has been outlined in recent models [3,4]. As a basic idea of these models, thermodynamically independent variables were extended by the set of fast variables. The dynamics of fast variables is consistent with the characteristic time of local rearrangement of particles (atoms or molecules) or with the relaxation time of diffusion flux to its local equilibrium steady-state value. In such a case, the dynamics of processes

\footnotetext{
* Corresponding author.

E-mail addresses: alex@ufn.ru (A. Olemskoi),peter.galenko@dlr.de (P. Galenko), alexandr.a.shmatko@univer.kharkov.ua (A. Shmatko).
}

can be described for large driving forces for diffusion, rapid cooling/heating conditions, large chemical gradients, or short periods of time.

A characteristic peculiarity of the theory of the diffusion process in rapid phase transitions $[3,4]$ is that time intervals are comparable with or even shorter than characteristic scale of diffusion flux variation. The latter appears together with a mixture concentration, as fluctuating independent variable. The role of the diffusion flux, therefore, has been analyzed in two descriptions [5]. First, the shortest observable time interval is shorter than the flux relaxation time, and diffusion flux behaves as fluctuating independent variable. Second, the shortest observable time interval is longer than the flux relaxation time, and the diffusion flux behaves as a Markovian hydrodynamic noise. This Letter is devoted to consideration of the second of these cases. Our approach is based on the supersymmetric field-theoretical scheme [6] which allows one to unify principle variables in such a manner that Bose-superfield components represent field variable and amplitude of fluctuations of diffusion flux, whereas the mutually conjugated Fermi-components give the mixture concentration.

Along this line, we set out in Section 2 main statements of the standard field-theoretical scheme based on the Langevin 
equation and the generating functional. Then, in Section 3 we consider the simplest case of the homogeneous concentration distribution. For this study we use dual fields that comprise of the Bose-components only. We show that two representations of such type are possible: the first of these leads to equations of motion with the first order time derivative, whereas the second one is represented by a Newtonian-type equation. The general case of the inhomogeneous concentration distribution is studied in Section 4 by introducing of the superfield Fermi-components, which give concentration and mean value of the diffusion flux. (In this way, Appendix A shows that supersymmetry theory renders main statements of standard theory of the diffusion process.) According to discussion in Section 5 the proposed supersymmetry approach allows one to obtain automatically fluctuation-dissipation relations for correlators of different components of superfield together with connection of the concentration and response of the order parameter onto diffusion flux. Moreover, we show that rotation in superspace gives connection of different components of superfield in equations of motion. In a case of the homogeneous concentration distribution, reduction of the superfield into a dual field is achieved due to fact that Grassmannian variables squared are equal to nilpotent one. Some extension of the present model to non-isothermal conditions is also considered. Conclusions are outlined in Section 6.

\section{Main statements}

Let us consider an isothermal system which is described by the Langevin equation for a stochastic field variable. This is

$\dot{x}(\mathbf{r}, t)-D \nabla^{2} x=-\gamma \frac{\partial f}{\partial x}+\zeta(\mathbf{r}, t)$,

where dot stands for the time derivative, $\nabla \equiv \partial / \partial \mathbf{r}, D$ is the diffusion coefficient, $\gamma$ is the kinetic coefficient, $f(x)$ is the specific free energy, $\zeta(\mathbf{r}, t)$ is the stochastic force defined by the white noise conditions:

$\langle\zeta(\mathbf{r}, t)\rangle=0, \quad\langle\zeta(\mathbf{r}, t) \zeta(\mathbf{0}, 0)\rangle=\gamma T \delta(\mathbf{r}) \delta(t)$.

In Eq. (2), angular brackets denote averaging over $\zeta(\mathbf{r}, t)$ and $T$ is the noise intensity being the temperature. With introducing measure units $t_{s} \equiv(\gamma T)^{2} / D^{3}, r_{s} \equiv \gamma T / D, f_{s} \equiv D^{3} / \gamma^{3} T^{2}$, $\zeta_{s} \equiv D^{3} /(\gamma T)^{2}$ for the time $t$, coordinate $\mathbf{r}$, specific free energy $f$ and stochastic force $\zeta$, respectively, Eq. (1) takes the simple form

$\dot{x}(\mathbf{r}, t)=-\frac{\delta \mathcal{F}}{\delta x}+\zeta(\mathbf{r}, t)$,

where short denotation of the variational derivative is used:

$\frac{\delta \mathcal{F}}{\delta x} \equiv \frac{\delta \mathcal{F}\{x(\mathbf{r}, t)\}}{\delta x(\mathbf{r}, t)}=\frac{\partial f}{\partial x}-\nabla^{2} x$

$$
\mathcal{F}\{x\} \equiv \int\left[f(x)+\frac{1}{2}(\nabla x)^{2}\right] \mathrm{d} \mathbf{r} .
$$

Our approach is based on the generating functional method [6]

$$
Z\{x(\mathbf{r}, t)\} \equiv\left\langle\prod_{(\mathbf{r}, t)} \delta\left\{\dot{x}+\frac{\delta \mathcal{F}}{\delta x}-\zeta\right\} \operatorname{det}\left|\frac{\delta \zeta}{\delta x}\right|\right\rangle
$$

which uses the Laplace representation of $\delta$-function as the functional integral over an ghost field $p(\mathbf{r}, t)$ :

$\delta\{y(\mathbf{r}, t)\}=\int \exp \left(-\int p y \mathrm{~d} \mathbf{r} \mathrm{d} t\right) D p(\mathbf{r}, t)$.

We show that the partition function can be represented in the following standard form

$$
\begin{gathered}
Z\{x(\mathbf{r}, t)\}=\int \exp [-\mathcal{S}\{x(\mathbf{r}, t), p(\mathbf{r}, t)\}] D p(\mathbf{r}, t), \\
\mathcal{S}\{x(\mathbf{r}, t), p(\mathbf{r}, t)\} \equiv \int L(x(\mathbf{r}, t), p(\mathbf{r}, t)) \mathrm{d} \mathbf{r} \mathrm{d} t,
\end{gathered}
$$

where $D p(\mathbf{r}, t)$ denotes the functional integration, the Lagrangian form $L(x, p)$ depends of the stochastic relation between principle field $x(\mathbf{r}, t)$ and white noise $\zeta(\mathbf{r}, t)$.

\section{Homogeneous concentration distribution}

Let us consider initially the simplest case, when the Jakobian of transition from the white noise $\zeta(\mathbf{r}, t)$ to the principle field $x(\mathbf{r}, t)$ takes the trivial form

$\operatorname{det}\left|\frac{\delta \zeta}{\delta x}\right|=1$

Then, using the Gaussian distribution for the white noise $\zeta(\mathbf{r}, t)$, we obtain the Lagrangian of the Euclidean field theory [7]

$L(x, p)=\left(p \dot{x}-\frac{1}{2} p^{2}\right)+p \frac{\delta f}{\delta x}$.

The Euler equations

$\dot{\Phi}=-\frac{\delta f}{\delta \Phi}+\mathrm{j}, \quad \mathrm{j}=\frac{\delta^{2} f}{\delta \Phi^{2}} \mathrm{j}$,

describe the evolution of the most probable values $\Phi \equiv x^{(\max )}$, $\mathrm{j} \equiv p^{(\max )}$ of the variable $x$ and the conjugate momentum $p$. The first of these equations shows explicitly that the quantity $\mathbf{J}$ plays the role of the most probable value of the diffusion flux related to the order parameter $\Phi$, whereas the ghost field $p$ is reduced to the fluctuating value of this flux.

Combination of Eqs. (10) leads to the following equation

$\ddot{\Phi}+\frac{\delta^{2} f}{\delta \Phi^{2}} \dot{\Phi}=\frac{\delta^{2} f}{\delta \Phi^{2}} \mathrm{~J}$.

It should be noted that in contrast to the common result obtained from the phase-field model $[3,4]$, Eq. (11) explicitly contains the diffusion flux as independent variable, whose evolution in time is determined by the second equation of (10).

With introducing a new field

$F \equiv \dot{x}-p$

the Lagrangian (9) takes the form

$L(x, f)=\frac{1}{2}\left(\dot{x}^{2}-F^{2}\right)-\frac{\delta f}{\delta x} F+\frac{\delta f}{\delta x} \dot{x}$, 
where the last term is considered as a total time derivative. Equations of motion are described by

$\ddot{\Phi}=-\frac{\delta^{2} f}{\delta^{2} \Phi} F, \quad F=-\frac{\delta f}{\delta \Phi}$.

The first equation has the Newtonian form, whereas the second one gives the definition of the thermodynamic force related to the order parameter. Insertion of definition (12) into the first of Eqs. (14) leads to the equation of motion (11).

Eqs. (10) and (14) may be obtained within the elegant supersymmetry-type scheme, whose usage will allow us to consider a general case of the inhomogeneous concentration distribution (see Section 4). With this aim, we introduce a dual field

$\phi_{p} \equiv x+\vartheta p$

where the nilpotent variable $\vartheta$ satisfies the conditions

$\vartheta^{2}=0, \quad \vartheta \vartheta^{\prime}=\vartheta^{\prime} \vartheta, \quad \int \mathrm{d} \vartheta=0, \quad \int \vartheta \mathrm{d} \vartheta=1$.

Then, the action appears as

$\mathcal{S}\{\phi(z)\}=\int \lambda(\phi(z)) \mathrm{d} z, \quad z \equiv\{\mathbf{r}, t, \vartheta\}$,

where the Lagrangian (9) takes the canonical form

$L=\int \lambda \mathrm{d} \vartheta, \quad \lambda(\phi) \equiv \frac{1}{2} \phi D_{p} \phi+f(\phi)$

with operator

$D_{p}=-\frac{\partial}{\partial \vartheta}+\left(1-2 \vartheta \frac{\partial}{\partial \vartheta}\right) \frac{\partial}{\partial t}$.

Being projected on the axes 1 and $\vartheta$, related Euler equation

$D \frac{\delta \lambda}{\delta(D \phi)}+\frac{\delta \lambda}{\delta \phi}=0 \Rightarrow D \phi+\frac{\delta f}{\delta \phi}=0$

arrives at equations of motion (10).

On the other hand, another dual field

$\phi_{F} \equiv x-\vartheta F$

accompanied with operator

$D_{F}=-\left(\frac{\partial}{\partial \vartheta}+\vartheta \frac{\partial^{2}}{\partial t^{2}}\right)$,

taken instead of definitions (15) and (19), get the equations of motion in the form (14). Characteristically, mutually inverted transformations of the dual fields (15) and (21) are achieved by means of the exponential differentiation operator [7]:

$\phi_{\mp F}(t)=\exp \left( \pm \vartheta \frac{\partial}{\partial t}\right) \phi_{\mp p}(t)$,

$\phi_{ \pm p}(t)=\exp \left( \pm \vartheta \frac{\partial}{\partial t}\right) \phi_{ \pm F}(t)$

These transformations are reduced into the formal shift $\vartheta$ of the time origin [7]:

$\phi_{\mp F}(t)=\phi_{\mp p}(t \pm \vartheta), \quad \phi_{ \pm p}(t)=\phi_{ \pm F}(t \pm \vartheta)$.

\section{Inhomogeneous concentration distribution}

Formal basis of the supersymmetry scheme is known to be the Jakobian representation [6]

$\operatorname{det}\left|\frac{\delta \zeta}{\delta x}\right|=\int \exp \left(\bar{q} \frac{\delta \zeta}{\delta x} q\right) \mathrm{d}^{2} q, \quad \mathrm{~d}^{2} q \equiv \mathrm{d} q \mathrm{~d} \bar{q}$,

where $q(\mathbf{r}, t)$ and $\bar{q}(\mathbf{r}, t)$ are the Grassmannian conjugated fields subjected to the following conditions

$\bar{q} q+q \bar{q}=0, \quad \int \mathrm{d} q=0$,

$\int q \mathrm{~d} q=1, \quad \int \mathrm{d} \bar{q}=0, \quad \int \bar{q} \mathrm{~d} \bar{q}=1$.

Then, the Laplace representation (6) leads to the supersymmetry Lagrangian

$\mathcal{L}(x, p, q, \bar{q})=\left(p \dot{x}-\frac{p^{2}}{2}+\frac{\delta f}{\delta x} p\right)-\bar{q}\left(\frac{\partial}{\partial t}+\frac{\delta^{2} f}{\delta x^{2}}\right) q$.

Introducing the four-component superfield

$\Psi_{p}=x+\bar{\theta} q+\bar{q} \theta+\bar{\theta} \theta p$,

it is easy to show that expression (27) can be reduced to canonical supersymmetry form

$\mathcal{L}=\int \Lambda \mathrm{d}^{2} \theta, \quad \mathrm{d}^{2} \theta \equiv \mathrm{d} \theta \mathrm{d} \bar{\theta}$,

$\Lambda\left(\Psi_{p}\right) \equiv \frac{1}{2}\left(\overline{\mathcal{D}}_{p} \Psi_{p}\right)\left(\mathcal{D}_{p} \Psi_{p}\right)+f\left(\Psi_{p}\right)$.

In this equation, the Grassmannian conjugate coordinates $\theta$ and $\bar{\theta}$ are defined by properties similar to Eqs. (26):

$\bar{\theta} \theta+\theta \bar{\theta}=0, \quad \int \mathrm{d} \theta=0$,

$\int \theta \mathrm{d} \theta=1, \quad \int \mathrm{d} \bar{\theta}=0, \quad \int \bar{\theta} \mathrm{d} \bar{\theta}=1$.

Supersymmetry generators in Eq. (29) are given by

$\mathcal{D}_{p}=\frac{\partial}{\partial \bar{\theta}}-2 \theta \frac{\partial}{\partial t}, \quad \overline{\mathcal{D}}_{p}=\frac{\partial}{\partial \theta}$.

The extremum condition of the superaction

$\mathcal{S}\{\Psi(\mathbf{r}, t)\}=\int \mathcal{L}(\Psi(\mathbf{r}, t)) \mathrm{d} t$

gives the supersymmetry Euler equation [7]

$-\frac{1}{2}[\overline{\mathcal{D}}, \mathcal{D}] \Psi+\frac{\delta f}{\delta \Psi}=0$,

where square brackets notice commutator. Taking the projections of Eq. (33) onto the axes $1, \bar{\theta}, \theta$ and $\bar{\theta} \theta$, we obtain the equations of motion in the following explicit form

$\dot{\Phi}-\nabla^{2} \Phi=-\frac{\partial f}{\partial \Phi}+\mathrm{j}$,

$\mathrm{j}+\nabla^{2} \mathbf{j}=\frac{\partial^{2} f}{\partial \Phi^{2}} \mathbf{j}-\frac{\partial^{3} f}{\partial \Phi^{3}} \bar{\psi} \psi$,

$\dot{\psi}-\nabla^{2} \psi=-\frac{\partial^{2} f}{\partial \Phi^{2}} \psi$ 
$-\dot{\bar{\psi}}-\nabla^{2} \bar{\psi}=-\frac{\partial^{2} f}{\partial \Phi^{2}} \bar{\psi}$

which define the most probable realizations of the fields $\Phi \equiv$ $x^{(\max )}, \mathrm{j} \equiv p^{(\max )}, \psi \equiv q^{(\max )}, \bar{\psi} \equiv \bar{q}^{(\max )}$. With $\psi=\bar{\psi}=0$, the first of these equations takes the form of the Langevin equation (3). This shows that ghost field $\mathrm{j} \equiv p^{(\max )}$ represents the most probable realization of the flux fluctuation amplitude $\zeta$. It is principally important that, in contrast to initial fields $x(\mathbf{r}, t)$, $p(\mathbf{r}, t), q(\mathbf{r}, t)$, and $\bar{q}(\mathbf{r}, t)$ being stochastic in its nature, the most probable fields $\Phi(\mathbf{r}, t), \mathbf{j}(\mathbf{r}, t), \psi(\mathbf{r}, t)$, and $\bar{\psi}(\mathbf{r}, t)$ are deterministic ones.

Combining the pair of Eqs. (36) and (37), we get the conservation law

$\dot{X}+\nabla \cdot \mathbf{J}=0$

for the quantities

$X \equiv \bar{\psi} \psi, \quad \mathbf{J} \equiv \nabla \bar{\psi} \cdot \psi-\bar{\psi} \cdot \nabla \psi$,

which define the concentration and the diffusion flux, respectively. As is shown in Appendix A, the flux (39) is reduced to Fick's law (66).

By analogy with the dual field (21), we can introduce the second supersymmetry representation

$\Psi_{F} \equiv x+\bar{\theta} \psi+\bar{\psi} \theta-\bar{\theta} \theta F$.

Then, the supersymmetry Lagrangian takes the form

$\mathcal{L}=\int \Lambda \mathrm{d}^{2} \theta, \quad \Lambda\left(\Psi_{F}\right) \equiv-\frac{1}{2} \Psi_{F} \overline{\mathcal{D}}_{F} \mathcal{D}_{F} \Psi_{F}+f\left(\Psi_{F}\right)$,

where the supersymmetry conjugated operators read as

$\mathcal{D}_{F}=\frac{\partial}{\partial \bar{\theta}}-\theta \frac{\partial}{\partial t}, \quad \overline{\mathcal{D}}_{F}=\frac{\partial}{\partial \theta}-\bar{\theta} \frac{\partial}{\partial t}$.

The explicit form of equations of motion

$\ddot{\Phi}=-\frac{\delta^{2} f}{\delta \Phi^{2}} F-\frac{\delta^{3} f}{\delta \Phi^{3}} \bar{\psi} \psi, \quad F=-\frac{\delta f}{\delta \Phi}$

is reduced to modified relations (14), being accompanied by Eqs. (36) and (37).

Similarly to the case of dual fields, transformations of the supersymmetry field (28) into the form of Eq. (40) are given by [cf. Eqs. (23) and (24)]:

$\Psi_{F}(t)=\exp \left(-\bar{\theta} \theta \frac{\partial}{\partial t}\right) \Psi_{p}(t), \quad \Psi_{F}(t)=\Psi_{p}(t-\bar{\theta} \theta)$.

\section{Discussion}

The principle advantage of the supersymmetry method is that it automatically derives fundamental relations between correlators of different superfield components. Such relations follow from the invariance condition of the superaction (32) under supersymmetry transformations. In this way, the fluctuation-dissipation theorem connects the structure factor $S(t) \equiv$ $\langle x(t) x(0)\rangle$ with advanced $G_{+}(t) \equiv\langle x(0) \zeta(t)\rangle$ and retarded $G_{-}(t) \equiv\langle x(t) \zeta(0)\rangle$ Green functions in the following manner [7]

$2 \dot{S}(t)=G_{+}(t)-G_{-}(t), \quad t>0$.
On the other hand, supersymmetry conditions lead to the following connection of different projections of supercorrelators on the superspace axes:

$\langle x(t) \zeta(0)\rangle=\langle\bar{\psi}(0) \psi(t)\rangle, \quad t>0$.

Taking into account the first of the definitions (39) and the simplest form of the fluctuation-dissipation relation $\langle x \zeta\rangle=\mathrm{d} \Phi / \mathrm{d}_{\mathrm{j}}$, one can obtain the important connection of the concentration with response of the order parameter onto the conjugated flux. At the time $t=0$, this yields

$X=\frac{\mathrm{d} \Phi}{\mathrm{d} \mathrm{j}}$.

According to Eqs. (46) and (47), the binary mixture concentration $X$ is determined by the change of the order parameter $\Phi$ with respect to the microflux $\mathrm{j}$. Being the most probable value of the fluctuation amplitude of the variable $x$, the microflux $\mathrm{j}$ is not reduced to the flux $\mathbf{J}$, which is defined by the second equation from Eqs. (39) to describe concentration variations over macroscopic distances, in accordance with Eq. (38).

Another advantage of the supersymmetry approach is that this method allows one to take into account connections of different superfield components in equation of motion (34) in a self-consistent manner. This is achieved as coupling supersymmetry components due to the supersymmetry transformation

$\tilde{\Psi}_{p} \equiv \mathrm{e}^{-\delta \bar{\theta} \psi} \Psi_{p}$,

which relates to a rotation in superspace fixed with parameter $\delta$. With $\delta \ll 1$, equations of motion take the following form [7]

$\dot{\Phi}-\nabla^{2} \Phi=-\frac{\partial f}{\partial \Phi}+\mathrm{j}+\delta \bar{\psi} \psi$

$\mathrm{j}+\nabla^{2} \mathrm{j}=\frac{\partial^{2} f}{\partial \Phi^{2}} \mathrm{j}-\frac{\partial^{3} f}{\partial \Phi^{3}} \bar{\psi} \psi-\delta \bar{\psi} \dot{\psi}$,

$\dot{\psi}-\nabla^{2} \psi=-\frac{\partial^{2} f}{\partial \Phi^{2}} \psi+\delta\left[(\dot{\Phi}-\mathrm{j})+\frac{\partial^{2} f}{\partial \Phi^{2}} \Phi\right] \psi$,

$\dot{\bar{\psi}}+\nabla^{2} \bar{\psi}=\frac{\partial^{2} f}{\partial \Phi^{2}} \bar{\psi}+\delta \Phi \dot{\bar{\psi}}$.

According to these equations not only the concentration $X$ affects the order parameter $\Phi$, but the latter essentially modifies the concentration variations as well. Thus, the combination of Eqs. (51) and (52) gives

$\dot{X}+\nabla \cdot \mathbf{J}=\delta\left(-\frac{\partial f}{\partial \Phi}+2 \frac{\partial^{2} f}{\partial \Phi^{2}} \Phi\right) X$

instead of the conservation law (38).

Now we show how the supersymmetry scheme is reduced to dual fields in the case of homogeneous concentration distribution. Using superfield (28), the kinetic energy in superLagrangian (29) can be reduced to the form

$-\frac{1}{4} \Psi_{p}\left[\overline{\mathcal{D}}_{p}, \mathcal{D}_{p}\right] \Psi_{p}$

where the commutator

$-\frac{1}{2}\left[\overline{\mathcal{D}}_{p}, \mathcal{D}_{p}\right]=-\frac{\partial^{2}}{\partial \theta \partial \bar{\theta}}+\left(1-2 \theta \frac{\partial}{\partial \theta}\right) \frac{\partial}{\partial t}$ 
is reduced to dual form (19) at condition $\vartheta \equiv \bar{\theta} \theta$ (i.e., Grassmannian variables squared are equal nilpotent one). In the case of superfield (40), supergenerators (42) in super-Lagrangian (41) get the combination

$$
-\overline{\mathcal{D}}_{f} \mathcal{D}_{f}=-\left(\frac{\partial}{\partial \theta} \frac{\partial}{\partial \bar{\theta}}+\bar{\theta} \theta \frac{\partial^{2}}{\partial t^{2}}\right)+\left(\bar{\theta} \frac{\partial}{\partial \bar{\theta}}-\theta \frac{\partial}{\partial \theta}\right) \frac{\partial}{\partial t},
$$

which is reduced to operator (22) at the following conditions

$\bar{\theta} \theta \equiv \vartheta, \quad \bar{\theta}(\partial / \partial \bar{\theta})=\theta(\partial / \partial \theta)$.

As a final note, the above consideration is related to isothermal conditions, when the temperature $T \equiv$ const is a parameter for the free energy $f(T)$. In non-isothermal situation, the thermodynamic state is defined through the specific internal energy $e$ fixing the specific entropy $s(e)$. Therefore, the passage from isothermal conditions to non-isothermal ones is achieved on the basis of the main thermodynamic identity

$f=e-T s(e, \Phi, X ; \mathbf{q}, \mathbf{J}, \mathbf{J})$,

where the set of slow classic variables $e, \Phi, X$ and related fluxes $\mathbf{q}, \mathbf{J}, \mathbf{J}$ is shown in the manner of Ref. [4]. The specific internal energy $e$ is determined by the conservation law

$\dot{e}+\nabla \cdot \mathbf{q}=0$

where the heat flux $\mathbf{q}$ is given by the following generalized relation [4]

$\mathbf{q}=\mathcal{M} \nabla\left(\frac{\partial s}{\partial e}+\varepsilon_{e}^{2} \nabla^{2} e\right)-\tau_{T} \dot{\mathbf{q}}$,

$\mathcal{M}$ being the mobility coefficient, $\varepsilon_{e}$ is the correlation spatial length, $\tau_{T}$ is the time for relaxation of the heat flux to its steady-state value. Combination of two last equations leads to the hyperbolic equation of motion for the specific internal energy:

$\tau_{T} \ddot{e}+\dot{e}=-\nabla \cdot\left[\mathcal{M} \nabla\left(\frac{\partial s}{\partial e}+\varepsilon_{e}^{2} \nabla^{2} e\right)\right]$.

In the simplest case of instant relaxation of the heat flux and vanishing spatial correlation, i.e., with $\tau_{T} \rightarrow 0$ and $\varepsilon_{e} \rightarrow 0$, one can obtain the following standard equation

$\dot{e}=-\nabla \cdot \mathcal{M} \nabla T^{-1}$,

which is a partial differential equation of a parabolic type.

\section{Conclusions}

A non-equilibrium binary system with diffusion has been considered. To analyze the diffusion, a special description for the diffusion flux has been taken into account. Namely, we analyzed the situation in which the shortest observable time interval is longer than the flux relaxation time, and the diffusion behaves as a Markovian hydrodynamic noise [5].

We used the idea that Bose-components of superfield are related to order parameter and amplitude of fluctuations of diffusion flux, while combination of the mutually conjugated Fermi-components gives the mixture concentration. A principle advantage of such a scheme is that the supersymmetry approach allows one to obtain automatically fluctuation-dissipation relations for correlators of different components of superfield. As a result, we obtain the fundamental connection (47) of the concentration and response of the order parameter with respect to the diffusion microflux. Thus, it was demonstrated that main equations of non-equilibrium binary system may be reproduced within the framework of stochastic supersymmetry field-theoretical scheme [7].

As a perspective, several generalizations of the present supersymmetry model can be outlined. First, a system in which the shortest observable time interval can be shorter than the flux relaxation time, and the diffusion flux behaves as fluctuating independent variable can be described within the supersymmetry model. Second, a system with both atomic diffusion and phase separation can be analyzed. And, third, a model's extension to non-isothermal binary system seems to be the natural advancement for the analysis.

\section{Acknowledgements}

P.G. acknowledges support by European Space Agency within the Research Programme "Life and Physical Sciences", project MSM-GA/2005-029.

\section{Appendix A}

It is easily to show that the second of definitions (39) gains the usual Fick's law. Really, within the wave representation one has

$\psi(\mathbf{r})=\sum_{\mathbf{k}} \psi_{\mathbf{k}} \exp (\mathrm{ikr}), \quad \bar{\psi}(\mathbf{r})=\sum_{\mathbf{k}} \psi_{\mathbf{k}} \exp (-\mathrm{ikr} \mathbf{r})$

Then, the gradients are

$\nabla \psi(\mathbf{r})=\sum_{\mathbf{k}}(\mathrm{ik}) \psi_{\mathbf{k}} \exp (\mathrm{ikr})$
$\nabla \bar{\psi}(\mathbf{r})=\sum_{\mathbf{k}}(-\mathrm{ik}) \bar{\psi}_{\mathbf{k}} \exp (-\mathrm{ik \mathbf {k }})$,

and the second of definitions (39) takes the form

$$
\begin{aligned}
\mathbf{J}= & -\sum_{\mathbf{k} \mathbf{k}^{\prime}}\left\{(\mathrm{ik}) \bar{\psi}_{\mathbf{k}} \psi_{\mathbf{k}^{\prime}} \exp \left[-\mathrm{i}\left(\mathbf{k}-\mathbf{k}^{\prime}\right) \mathbf{r}\right]\right. \\
& \left.+(\mathrm{ik}) \bar{\psi}_{\mathbf{k}^{\prime}} \psi_{\mathbf{k}} \exp \left[\mathrm{i}\left(\mathbf{k}-\mathbf{k}^{\prime}\right) \mathbf{r}\right]\right\} .
\end{aligned}
$$

After renaming $\mathbf{k}$ into $-\mathbf{k}^{\prime}$ and vice versa in the second term, the last expression can be written as the derivative

$\mathbf{J}=-\nabla X$

where in accordance with Eqs. (39) and (63) the concentration is represented as the wave expansion:

$X(\mathbf{r})=\sum_{\mathbf{k} \mathbf{k}^{\prime}} \bar{\psi}_{\mathbf{k}} \psi_{\mathbf{k}^{\prime}} \exp \left[\mathrm{i}\left(\mathbf{k}-\mathbf{k}^{\prime}\right) \mathbf{r}\right] \equiv \bar{\psi}(\mathbf{r}) \psi(\mathbf{r})$.

Thus, the supersymmetry field definitions (39) give Fick's law (66) (recall that we use dimensionless units where the diffusion coefficient is hidden within a space scale). 


\section{References}

[1] D. Jou, J. Casas-Vazquez, G. Lebon, Rep. Prog. Phys. 51 (1988) 1005.

[2] D. Jou, J. Casas-Vazquez, G. Lebon, Rep. Prog. Phys. 62 (1999) 1035.

[3] P. Galenko, Phys. Lett. A 287 (2001) 190.

[4] P. Galenko, D. Jou, Phys. Rev. E 71 (2005) 046125.
[5] D. Jou, P. Galenko, Physica A 366 (2006) 149.

[6] J. Zinn-Justin, Quantum Field Theory and Critical Phenomena, Clarendon, Oxford, 1993.

[7] A.I. Olemskoi, Phys. Usp. 44 (5) (2001) 479. 